\title{
Elasmobranch species in the artisanal fishery of Sucre State, Venezuela
}

\section{Especies de elasmobranquios en la pesca artesanal del estado Sucre, Venezuela}

\author{
Raquel Marquez ${ }^{1 *}$, Rafael Tavares ${ }^{2}$, Luis Alejandro Ariza ${ }^{3}$ \\ 1 Departamento de Biología, Universidad de Oriente, Núcleo Sucre, Cumaná, Venezuela. \\ 2 Centro para la Investigación de Tiburones, Distrito Capital, Caracas, Venezuela. \\ ${ }^{3}$ Laboratorio de Biología Pesquera, Instituto Oceanográfico de Venezuela, Cumaná, Venezuela.
}

* Corresponding author. E-mail: rvmarquez94@gmail.com

\begin{abstract}
A wide diversity of elasmobranch species has been reported for waters off Venezuela; however, information regarding this group is lacking for most coastal regions. This study aimed to determine the species composition in catches made by the artisanal fishery of Sucre State, Venezuela, and to analyze size structure by sex for the most common species. During the study period (January 2016-September 2017), visits were made to the main fish markets in the city of Cumaná, Sucre State, and a total of 2,167 elasmobranchs belonging to 24 species (15 sharks and 9 rays) were quantified. The most frequently captured elasmobranch species were Mustelus higmani (36.2\%), Mustelus canis (18.0\%), Hypanus guttatus (15.1\%), and Hypanus americanus (7.1\%). The size ranges for these species were 29.3-73.7 cm total length (TL), 34.0-86.0 $\mathrm{cm}$ TL, 17.0-138.0 cm disc diameter (DD), and 48.0-108.0 cm DD, respectively. Considering national conservation assessments (following International Union for Conservation of Nature criteria), 7 of the species reported for the study area (M. higmani, Carcharhinus limbatus, Carcharhinus falciformis, Isurus oxyrinchus, Alopias superciliosus, Prionace glauca, and Aetobatus narinari) are listed as vulnerable, and the remaining species $(n=17)$ have not been assessed. Current conservation efforts in Venezuela are deficient given the collapse of government institutions responsible for the management of fisheries. This has led to noncompliance with fishing regulations designed for elasmobranchs. In the future, great effort must be made to continue generating information; however, future advances in scientific research and conservation will depend largely on the recovery of the economic and political stability in Venezuela.
\end{abstract}

Key words: biology, Caribbean, species, rays, sharks.

RESUMEN. En Venezuela existe una importante diversidad de especies de elasmobranquios. Sin embargo, existe escasa información con relación a estos en la mayor parte de las regiones costeras. Los objetivos del presente estudio fueron determinar la composición de especies en la captura de la pesca artesanal del estado Sucre, Venezuela, y analizar la estructura de tallas por sexo para las especies más comunes. Durante el periodo de estudio (enero de 2016-septiembre de 2017) se realizaron visitas a las principales áreas de comercialización de la ciudad de Cumaná, estado de Sucre, y se cuantificaron un total de 2,167 elasmobranquios pertenecientes a 24 especies (15 tiburones y 9 rayas). Las especies de elasmobranquios más frecuentemente capturadas fueron Mustelus higmani (36.2\%), Mustelus canis (18.0\%), Hypanus guttatus (15.1\%) y Hypanus americanus (7.1\%). Los intervalos de tallas (longitud total [LT], diámetro de disco [DD]) registrados para estas especies fueron 29.3-73.7 cm LT, 34.0-86.0 cm LT, 17.0-138.0 cm DD y 48.0-108.0 cm DD, respectivamente. Según las evaluaciones de conservación a nivel nacional (criterios de la Unión Internacional para la Conservación de la Naturaleza), 7 de las especies registradas en el área de estudio (M. higmani, Carcharhinus limbatus, Carcharhinus falciformis, Isurus oxyrinchus, Alopias superciliosus, Prionace glauca y Aetobatus narinari) se encuentran en estado vulnerable, mientras que el resto de las especies $(n=17)$ no han sido evaluadas. En Venezuela, actualmente, existe una gran deficiencia en materia de conservación debido al colapso de las instituciones encargadas de la administración pesquera. Ello conlleva el incumplimiento de las normativas de pesca diseñadas para los elasmobranquios. En el futuro se deberá hacer un gran esfuerzo para continuar generando información; sin embargo, los avances futuros en materia de investigación científica y conservación dependerán, en gran medida, de la recuperación de la estabilidad económica y política del país.

Palabras clave: biología, Caribe, especies, rayas, tiburones.

\section{INTRODUCTION}

Elasmobranchs are a taxonomic group that comprises sharks and rays (Nelson et al. 2016). Around 509 sharks species and 630 ray species are currently known to exist in a wide variety of marine and freshwater ecosystems worldwide (Weigmann 2016). Sharks and rays are particularly vulnerable to overexploitation due to their $K$-selected life history strategy and high natural mortality rates (Stevens et al. 2000). Recent assessments indicate that approximately $24 \%$ of elasmobranchs are threatened by potentially high extinction risks

\section{INTRODUCCIÓN}

Los elasmobranquios son un grupo de peces que taxonómicamente engloba a los tiburones y las rayas (Nelson et al. 2016). Actualmente, se conocen a nivel global alrededor de 509 especies de tiburones y 630 especies de rayas, las cuales habitan en diversos ecosistemas marinos y de agua dulce (Weigmann 2016). Los tiburones y las rayas son particularmente vulnerables a la sobreexplotación por sus características de historia de vida tipo $K$ y por sus elevadas tasas de mortalidad natural (Stevens et al. 2000). Las evaluaciones 
(Dulvy et al. 2014), overfishing being the main cause of population biomass declines (Baum et al. 2003, Baum and Myers 2004, Clarke et al. 2007).

A wide diversity of marine elasmobranchs has been reported for Venezuela, with a total of 66 shark species and 49 ray species, which represent about $61 \%$ of all species reported for the Western Central Atlantic (Tavares 2019). However, the health status of the most important populations is unknown because quantitative stock assessments have not been carried out. This knowledge deficiency is the consequence of partly the lack of biological and fishery data such as biological parameter estimates, relative abundance indices, amount of fishing effort, and fishery production databases. On the other hand, sharks and rays constitute a traditional fishery resource used throughout most of Venezuela's shoreline and islands, primarily via artisanal fishing activities. According to national fisheries production data, shark catches are almost entirely made by artisanal fisheries (94\%) and the rest by industrial fisheries (Tavares 2019), underpinning the impact and importance of artisanal fisheries in the country.

The northeast region of Venezuela, where Sucre State is located, is one of the country's most important fishing areas (Novoa et al. 1998). Moreover, this region is characterized by high marine productivity as a result of coastal upwelling influence (Margalef 1969, Castellanos et al. 2002). Elasmobranch landings in this region consist almost entirely $(\sim 80 \%)$ of sharks (Tavares 2019). Despite the significance of this fishery resource for the region, no inventories of the most frequently caught and marketed elasmobranch species have been made for marine and coastal areas off Sucre State. Therefore, the aim of this study was to determine species catch composition and to analyze population structure by sex for the most common species in the artisanal elasmobranch fishery.

\section{MATERIALS AND METHODS}

Although Venezuela's main artisanal and industrial fleets are based in the state of Sucre (Novoa et al. 1998), most elasmobranch catches marketed in the state are made by artisanal fishing. The fishing gear that is commonly used in artisanal fisheries to capture sharks and rays are gillnets and longlines (bottom and pelagic). Data were collected between January 2016 and September 2017 during visits $(n=29)$ to the fishing port and the municipal market of the city of Cumaná (capital of Sucre State), both the main marketplaces for artisanal fisheries.

Elasmobranchs were quantified and identified to the species taxonomic level using the taxonomic procedures available in the literature (Cervigón and Alcalá 1999, Compagno 2002, McEachran and de Carvalho 2002). When possible, the size (total length [TL, cm] for sharks and disc diameter [DD, $\mathrm{cm}$ ] for rays) and sex of each examined animal were recorded. Because most sharks were eviscerated and beheaded before arriving at the fishing ports, additional length measurements were recorded when complete animals were found: modified interdorsal length (first dorsal fin [1D] to second dorsal fin más recientes indican que aproximadamente el $24 \%$ de los elasmobranquios se encuentran amenazados con riesgo de extinción potencialmente elevado (Dulvy et al. 2014), y la sobrepesca es la principal causa de la disminución de la biomasa de las poblaciones (Baum et al. 2003, Baum y Myers 2004, Clarke et al. 2007).

En Venezuela existe una diversidad importante de elasmobranquios marinos, ya que se ha reportado un total de 66 especies de tiburones y 49 especies de rayas, lo que representa alrededor del $61 \%$ de todas las especies reportadas para el Atlántico centro-occidental (Tavares 2019). Sin embargo, no se conoce con exactitud el estado de salud de las poblaciones más importantes debido a que no ha sido posible realizar las evaluaciones pesqueras de manera cuantitativa. En parte, esta deficiencia de conocimiento ha sido consecuencia de la falta de información biológica y pesquera como estimaciones de parámetros biológicos, índices de abundancia relativa, niveles de esfuerzo pesquero y datos de producción. Por otra parte, los tiburones y las rayas constituyen un recurso pesquero tradicional que se aprovecha en casi toda la costa y las islas venezolanas, principalmente a través de actividades pesqueras artesanales. Con base en los datos de las estadísticas de producción pesquera nacional, las capturas de tiburones se originan casi en su totalidad de la pesca artesanal (94\%) y el resto procede de la pesca industrial (Tavares 2019), lo que muestra el impacto y la importancia que pueden tener las pesquerías artesanales en el país.

La región nororiental de Venezuela, donde se sitúa el estado Sucre, es una de las zonas pesqueras más importantes del país (Novoa et al. 1998). Además, esta región se caracteriza por poseer una elevada productividad marina como consecuencia de la influencia del fenómeno de surgencia costera (Margalef 1969, Castellanos et al. 2002). Los desembarques de elasmobranquios en esta región están constituidos casi en su totalidad $(\sim 80 \%)$ por tiburones (Tavares 2019$)$. A pesar de la relevancia de este recurso pesquero para la región, en las zonas marino-costeras del estado de Sucre no se había realizado un inventario de las especies de elasmobranquios más frecuentemente capturadas y comercializadas. En este sentido, los objetivos del presente estudio fueron determinar la composición de la captura por especies y analizar la estructura de tallas por sexo para las especies de elasmobranquios más comunes en la pesquería artesanal.

\section{MATERIALES Y MÉTODOS}

Si bien el estado de Sucre alberga parte de las principales flotas de las pesquerías artesanal e industrial en Venezuela (Novoa et al. 1998), las capturas de elasmobranquios que se comercializan en el estado provienen, en su mayoría, de la pesca artesanal. Los artes de pesca comúnmente utilizados en las pesquerías artesanales para la captura de tiburones y rayas son las redes de ahorque o agalleras y los palangres (de fondo y pelágicos). Los datos se recolectaron entre enero de 2016 y septiembre de 2017 durante visitas $(n=29)$ al puerto 
[2D], 1D-2D) and length from the pectoral fin (PC) (Fig. 1). This allowed reconstructing TLs for incomplete sharks and for the 2 most important species with the following conversion equations:

$$
\begin{gathered}
\text { Mustelus higmani, }(n=133): \\
\mathrm{TL}=10.09+2.38 \times 1 \mathrm{D}-2 \mathrm{D}\left(r^{2}=0.83\right) \\
\mathrm{TL}=3.94+1.17 \times \mathrm{PC}\left(r^{2}=0.89\right) \\
\text { Mustelus canis }(n=63): \\
\mathrm{TL}=8.58+2.47 \times 1 \mathrm{D}-2 \mathrm{D}\left(r^{2}=0.91\right) \\
\mathrm{TL}=5.92+1.11 \times \mathrm{PC}\left(r^{2}=0.92\right)
\end{gathered}
$$

Species composition of shark catches was given as a numerical percentage of the total number of species recorded. Sex and size data were analyzed in detail for the 4 elasmobranch species that were most frequently captured in the study area. A chi-square $\left(\chi^{2}\right)$ test was used to evaluate sex ratio $(M: F)$ and to detect whether there were statistical differences in male and female abundances (Zar 1996). Size structure was represented in frequency histograms by grouping individuals into $10-\mathrm{cm}$ (TL/DD) size classes. In addition, the categorical description of each species in the International

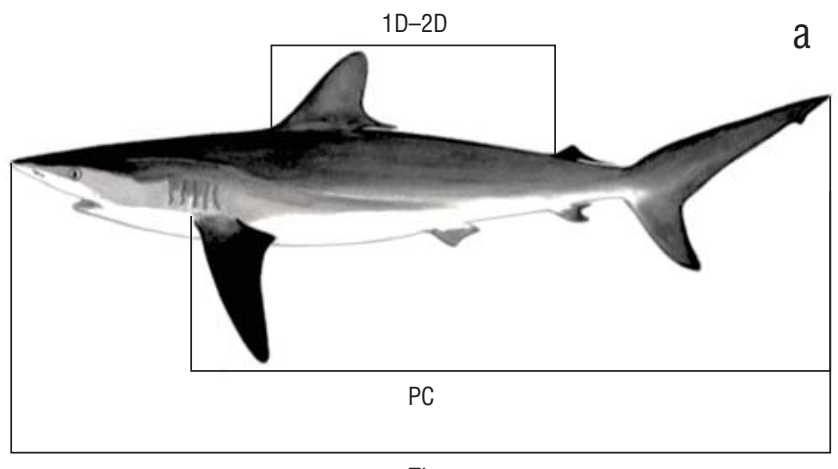

$\mathrm{TL}$

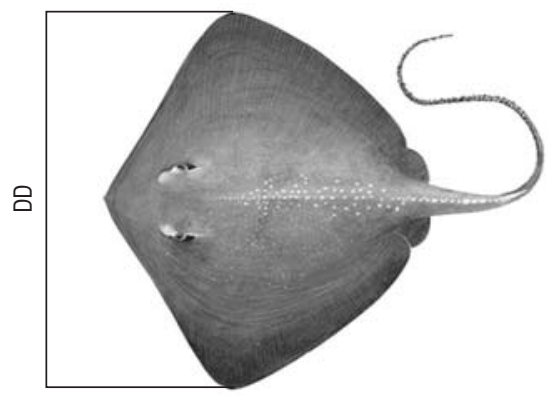

Figure 1. Morphometric measurements used for sharks (a) and rays (b) captured in commercial fisheries of the state of Sucre, Venezuela. 1D-2D, modified interdorsal length; PC, length from the pectoral fin; TL, total length; DD, disc diameter.

Figura 1. Medidas morfométricas de tiburones (a) y rayas (b) capturados en pesquerías comerciales del estado de Sucre, Venezuela. 1D-2D, longitud interdorsal modificada; PC, longitud desde la aleta pectoral; TL, longitud total; DD, diámetro del disco. pesquero y al mercado municipal de la ciudad de Cumaná (capital del estado de Sucre), que son los principales centros de acopio para la pesca artesanal.

Los elasmobranquios capturados fueron cuantificados e identificados a nivel taxonómico de especie utilizando los procedimientos taxonómicos disponibles en la literatura (Cervigón y Alcalá 1999, Compagno 2002, McEachran y de Carvalho 2002). En la medida de lo posible, a cada animal examinado se le registró la talla (longitud total [LT, cm] en los tiburones y el diámetro del disco [DD, cm] en las rayas) y el sexo. La mayoría de los tiburones llegan a los puertos de desembarque eviscerados y sin cabeza y, en ocasiones, solo es posible examinar el tronco de los individuos. En este sentido, para los animales completos, se registraron adicionalmente las siguientes medidas de talla: longitud interdorsal modificada (de primera aleta dorsal [1D] a segunda aleta dorsal [2D], 1D-2D) y longitud total desde la aleta pectoral (PC) (Fig. 1). Ello permitió la reconstrucción de las LT de los tiburones incompletos y de las 2 especies más importantes a través de las siguientes ecuaciones de conversión:

$$
\begin{gathered}
\text { Mustelus higmani, }(n=133): \\
\mathrm{LT}=10.09+2.38 \times 1 \mathrm{D}-2 \mathrm{D}\left(r^{2}=0.83\right) \\
\mathrm{LT}=3.94+1.17 \times \mathrm{PC}\left(r^{2}=0.89\right) \\
\text { Mustelus canis }(n=63): \\
\mathrm{LT}=8.58+2.47 \times 1 \mathrm{D}-2 \mathrm{D}\left(r^{2}=0.91\right) \\
\mathrm{LT}=5.92+1.11 \times \mathrm{PC}\left(r^{2}=0.92\right)
\end{gathered}
$$

Las capturas por especie se expresaron en porcentaje numérico del total del número de especies registradas. Para las 4 especies más frecuentemente capturadas en el área de estudio, los datos de sexo y talla fueron analizados más detalladamente. La proporción de sexos $(\mathrm{M}: \mathrm{H})$ se evaluó aplicando una prueba de chi cuadrado $\left(\chi^{2}\right)$ (Zar 1996) con el propósito de detectar si existía diferencia estadística en la abundancia entre machos y hembras. La estructura de tallas fue representada mediante histogramas de frecuencia agrupando a los individuos en clases de talla de $10 \mathrm{~cm}$ (LT/DD). Adicionalmente, se usó la categoría de cada especie en la lista roja de la Unión Internacional para la Conservación de la Naturaleza (UICN) (IUCN 2019) y en el Libro Rojo de la Fauna Venezolana (Tavares 2015) con la finalidad de conocer los niveles de amenaza a nivel global y a nivel nacional para las especies reportadas para el área de estudio.

\section{RESUltados}

Durante el periodo de estudio, se cuantificaron un total de 2,167 elasmobranquios pertenecientes a 24 especies, de las cuales 15 fueron tiburones y 9 fueron rayas (Tabla 1). El análisis de los datos pesqueros indicó que las especies más importantes en la composición de la captura fueron los tiburones M. higmani (36.2\%) y M. canis (18.0\%), seguidos de las rayas Hypanus guttatus (15.1\%) y Hypanus americanus (7.1\%). Otras especies relativamente importantes en la pesquería 
Union for Conservation of Nature (IUCN) Red List of Threatened Species (IUCN 2019) and in the Libro Rojo de la Fauna Venezolana (Red Book of Venezuelan Fauna) (Tavares 2015) was used to determine the global and national risk levels for the species reported in the study area.

\section{RESULTS}

During the study period, a total of 2,167 elasmobranchs were quantified and grouped into 24 species, 15 of which were sharks and 9 rays (Table 1). Analysis of fishery data indicated that the most important species in the catch composition were the sharks M. higmani (36.2\%) and M. canis $(18.0 \%)$, followed by the rays Hypanus guttatus (15.1\%) and Hypanus americanus (7.1\%). Other relatively important species in the elasmobranch fishery were Rhizoprionodon lalandii (6.5\%), Fontitrygon geijskesi (4.0\%), and Carcharhinus porosus (3.1\%).

The sex ratio observed in $M$. higmani catches (1.0:0.2) showed a highly significant predominance of males compared to females $\left(\chi^{2}=46.1 ; P<0.000\right)$. Male sizes ranged from 31.9 to $61.2 \mathrm{~cm} \mathrm{TL}$, and female sizes ranged from 29.3 to $73.7 \mathrm{~cm}$ TL (Fig. 2). For M. canis, the sex ratio (1.0:0.5) indicated that males were also significantly predominant in the catches $\left(\chi^{2}=\right.$ $10.1 ; P=0.002)$. Regarding sizes for this species, males measured between 36.4 and $86.0 \mathrm{~cm}$ TL, and females measured between 34.0 and $66.8 \mathrm{~cm}$ TL (Fig. 2).

The sex ratio for $H$. guttatus $(0.9: 1.0)$ showed no significant difference between males and females $\left(\chi^{2}=0.1, P=\right.$ 0.762 ). Male sizes ranged from 42.0 to $138.0 \mathrm{~cm} \mathrm{DD}$, and female sizes ranged from 17.0 to $118.0 \mathrm{~cm}$ DD (Fig. 2). For $H$. americanus, the sex ratio (0.9:1.0) also revealed no statistical difference between males and females $\left(\chi^{2}=0.1 ; P=\right.$ 0.787). Sizes for this species ranged from 49.0 to $100.0 \mathrm{~cm} \mathrm{DD}$ in males and from 48.0 to $108.0 \mathrm{~cm}$ DD in females (Fig. 2).

Following IUCN criteria, global conservation assessments indicate that 3 of the species recorded in this study are threatened to some level (Table 1). Isurus oxyrinchus is listed as endangered and Alopias superciliosus and Carcharhinus falciformis are listed as vulnerable. The rest of the species are listed as near threatened $(n=10)$, data deficient $(n=7)$, and least concern $(n=4)$. National conservation assessments indicate that 7 of the species caught in the study area (M. higmani, Carcharhinus limbatus, C. falciformis, I. oxyrinchus, A. superciliosus, Prionace glauca, and Aetobatus narinari) are categorized as vulnerable (Table 1). The rest of the species $(n=17)$ have not been evaluated at the national level because the technical and scientific information needed to adjust their conservation status is missing; therefore, the population health status of these species is currently unknown.

\section{Discussion}

The most common elasmobranchs in the study area were the sharks $M$. higmani and $M$. canis and the rays $H$. guttatus fueron Rhizoprionodon lalandii (6.5\%), Fontitrygon geijskesi (4.0\%) y Carcharhinus porosus (3.1\%).

La proporción sexual observada en las capturas de M. higmani (1.0:0.2) mostró una predominancia altamente significativa de los machos con respecto a las hembras $\left(\chi^{2}=\right.$ $46.1, P<0.000$ ). Las tallas de los machos oscilaron entre 31.9 y $61.2 \mathrm{~cm} \mathrm{LT}$, y las tallas de las hembras variaron entre 29.3 y $73.7 \mathrm{~cm}$ LT (Fig. 2). Con relación a M. canis, la proporción sexual (1.0:0.5) indicó que los machos también predominaron significativamente $\left(\chi^{2}=10.1, P=0.002\right)$ en las capturas examinadas. Con respecto a las tallas de esta especie, los machos midieron entre 36.4 y $86.0 \mathrm{~cm}$ LT y las hembras midieron entre 34.0 y $66.8 \mathrm{~cm}$ LT (Fig. 2).

La proporción sexual registrada para $H$. guttatus $(0.9: 1.0)$ no mostró diferencia significativa entre machos y hembras $\left(\chi^{2}=0.1, P=0.762\right)$. Las tallas de los machos variaron entre 42.0 y $138.0 \mathrm{~cm}$ DD y las de las hembras, entre $17.0 \mathrm{y}$ $118.0 \mathrm{~cm}$ DD (Fig. 2). En el caso de H. americanus, la proporción sexual (0.9:1.0) observada, igualmente, reveló que no hubo diferencia estadística entre la proporción de sexos $\left(\chi^{2}=\right.$ $0.1, P=0.787)$. Las tallas de esta especie oscilaron entre 49.0 y $100.0 \mathrm{~cm}$ DD para los machos y entre 48.0 y $108.0 \mathrm{~cm}$ DD para las hembras (Fig. 2).

De acuerdo con las evaluaciones de conservación a nivel global, según los criterios de la UICN, 3 de las especies registradas en el presente estudio se encuentran categorizadas con algún nivel de amenaza (Tabla 1). Isurus oxyrinchus está catalogada como en peligro y Alopias superciliosus y Carcharhinus falciformis están catalogadas bajo el estatus de vulnerable. El resto de las especies están clasificadas en las categorías de casi amenazadas $(n=10)$, datos insuficientes $(n=7)$ y preocupación menor $(n=4)$. A nivel nacional, las evaluaciones de conservación mostraron que 7 de las especies registradas en el área de estudio (M. higmani, Carcharhinus limbatus, C. falciformis, I. oxyrinchus, A. superciliosus, Prionace glauca y Aetobatus narinari) se encuentran bajo el estatus de vulnerable (Tabla 1). No hubo una evaluación nacional registrada para el resto de las especies $(n=17)$ debido a la insuficiencia de información técnico-científica necesaria para ajustar los niveles de su estatus de conservación; por lo tanto, se desconoce el estado de salud de sus poblaciones.

\section{Discusión}

Los elasmobranquios más comunes en el área de estudio fueron los tiburones $M$. higmani y $M$. canis y las rayas H. guttatus y H. americanus. Para la pesca de elasmobranquios realizada desde la isla de Margarita, adyacente al estado Sucre, Tavares et al. (2010) reportaron que las capturas en número estuvieron principalmente constituidas por M. higmani (40.8\%), Rhizoprionodon porosus (12.6\%) y $R$. lalandii (9.4\%). En otro estudio realizado frente a la isla de Cubagua, también localizada en la región nororiental, Cordovés et al. (2010) encontraron que las rayas más frecuentemente capturadas fueron $H$. guttatus (48.9\%), Myliobatis freminvillei 
and H. americanus. For the elasmobranch fishery in Margarita Island, adjacent to Sucre State, Tavares et al. (2010) reported that catches consisted mainly of $M$. higmani (40.8\%), Rhizoprionodon porosus (12.6\%), and R. lalandii (9.4\%). In a different study carried out off Cubagua, also located in the northeastern region, Cordovés et al. (2010) found that the most frequently captured ray species were $H$. guttatus (48.9\%), Myliobatis freminvillei (18.0\%), and H. americanus (15.7\%). However, further to the east, between Trinidad and Tobago and Guyana, the most important species in commercial landings were R. lalandii and C. porosus (Shing 2006, Kolmann et al. 2017). Altogether, these results show that sharks belonging to the genus Mustelus and rays belonging to the genus Hypanus are important elasmobranch groups in the northeastern region of Venezuela.
(18.0\%) y H. americanus (15.7\%). Por otra parte, más hacia la zona oriental, entre Trinidad y Tobago, y Guyana, las especies más importantes en las capturas comerciales fueron R. lalandii y C. porosus (Shing 2006, Kolmann et al. 2017). Estos resultados, en su conjunto, muestran que los tiburones del género Mustelus y las rayas del género Hypanus constituyen grupos de elasmobranquios importantes en la región nororiental de Venezuela.

Los resultados mostraron que para las especies M. higmani y $M$. canis hubo una amplia dominancia de ejemplares machos con relación a las hembras, pero también indican diferencias con relación a la estructura de tallas de los ejemplares según el sexo. Esta discrepancia entre sexos puede estar relacionada con diferencias en algunas variables biológicas, como los parámetros del crecimiento (Cortés et al. 2012). Estudios

Table 1. Species composition (\%) of elasmobranchs captured in commercial fisheries of the state of Sucre, Venezuela. Global (IUCN 2019) and national (Tavares 2015) conservation status: endangered (EN), vulnerable (VU), near threatened (NT), least concern (LC), data deficient (DD), and not evaluated (NE).

Tabla 1. Composición de la captura por especies (\%) para los elasmobranquios capturados en la pesca comercial del estado Sucre, Venezuela. Estatus de conservación global (IUCN 2019) y nacional (Tavares 2015): en peligro (EN), vulnerable (VU), casi amenazada (NT), preocupación menor (LC), datos insuficientes (DD) y no evaluada (NE).

\begin{tabular}{|c|c|c|c|c|c|c|}
\hline \multirow[t]{2}{*}{ Species } & \multirow[t]{2}{*}{ Common name in English } & \multirow[t]{2}{*}{ Common name in Spanish } & \multirow{2}{*}{$\begin{array}{l}\text { Number } \\
\text { (n) }\end{array}$} & \multirow{2}{*}{$\begin{array}{c}\text { Percentage } \\
(\%)\end{array}$} & \multicolumn{2}{|c|}{ Conservation status } \\
\hline & & & & & Global & National \\
\hline Mustelus higmani & Smalleye smoothhound & Viuda virma amarilla & 784 & 36.2 & $\mathrm{LC}$ & VU \\
\hline Hypanus guttatus & Longnose stingray & Raya látigo hocicona & 328 & 15.1 & DD & NE \\
\hline Hypanus americanus & Southern stingray & Raya americana & 154 & 7.1 & DD & NE \\
\hline Fontitrygon geijskesi & Sharpsnout stingray & Raya látigo picúa & 86 & 4.0 & NT & NE \\
\hline Carcharhinus porosus & Smalltail shark & Cazón poroso & 68 & 3.1 & DD & NE \\
\hline Myliobatis goodei & Southern eagle ray & Chucho amarillo & 52 & 2.4 & DD & NE \\
\hline Myliobatis freminvillei & Bullnose eagle ray & Chucho blanco & 51 & 2.4 & DD & NE \\
\hline Rhizoprionodon porosus & Caribbean sharpnose shark & Cazón playón & 50 & 2.3 & $\mathrm{LC}$ & NE \\
\hline Prionace glauca & Blue shark & Tiburón Azul & 6 & 0.3 & NT & VU \\
\hline Aetobatus narinari & Spotted eagle ray & Chucho pintado & 6 & 0.3 & NT & VU \\
\hline Galeocerdo cuvier & Tiger shark & Tiburón tigre & 4 & 0.2 & NT & $\mathrm{NE}$ \\
\hline Carcharhinus perezii & Caribbean reef shark & Tiburón piedrero & 2 & 0.1 & NT & $\mathrm{NE}$ \\
\hline Gynglimostoma cirratum & Nurse shark & Tiburón gata & 2 & 0.1 & DD & $\mathrm{NE}$ \\
\hline Alopias superciliosus & Bigeye thresher & Tiburón zorro ojón & 1 & 0.0 & VU & VU \\
\hline Carcharhinus falciformis & Silky shark & Tiburón bobo & 1 & 0.0 & VU & VU \\
\hline Heptranchias perlo & Sharpnose sevengill shark & Tiburón de fondo & 1 & 0.0 & NT & NE \\
\hline
\end{tabular}



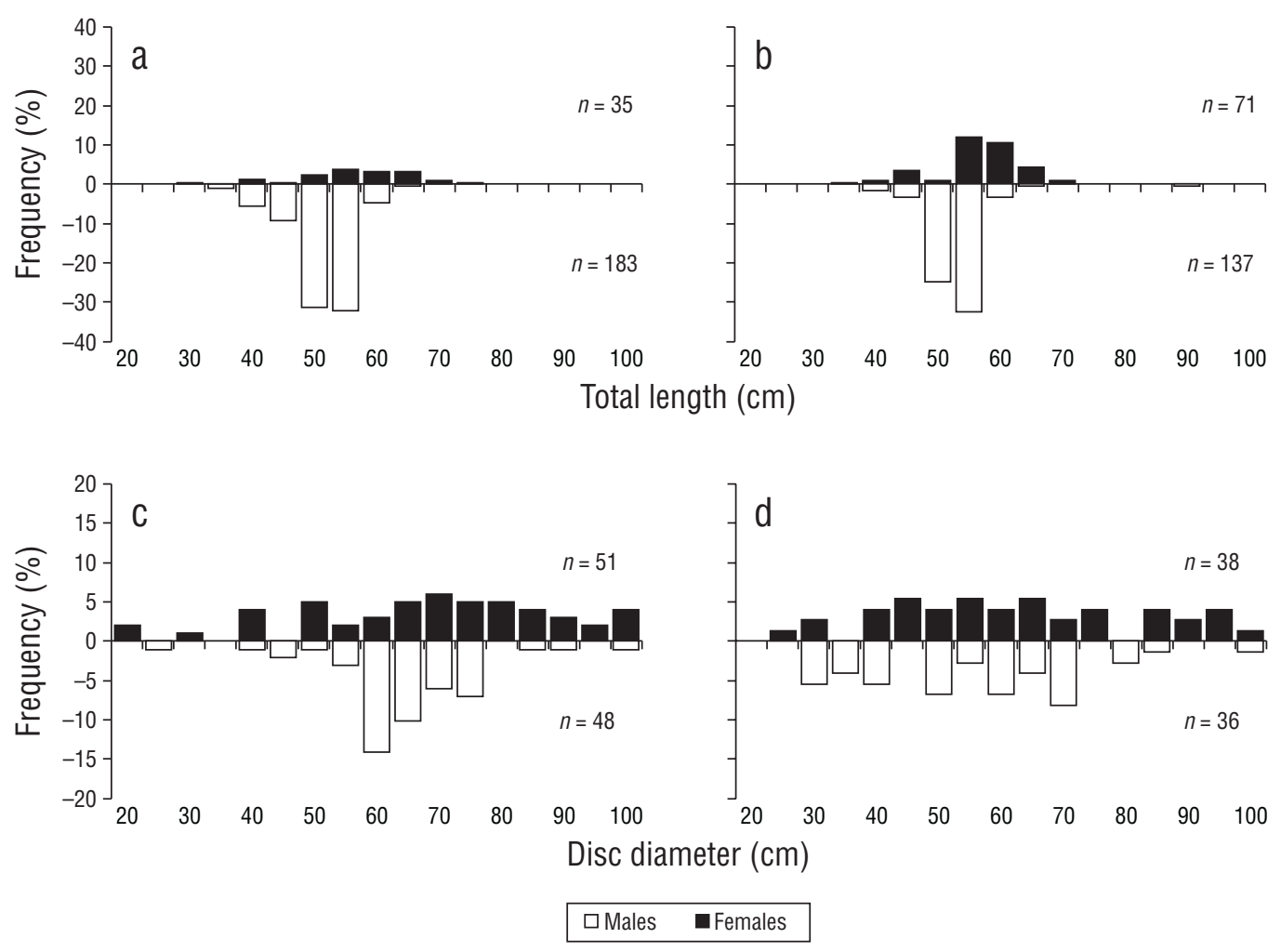

Figure 2. Sex-based size structure for the 4 most common elasmobranch species captured in commercial fisheries of the state of Sucre, Venezuela. Mustelus higmani (a), Mustelus canis (b), Hypanus guttatus (c), and Hypanus americanus (d).

Figura 2. Estructura de tallas por sexo para las 4 especies de elasmobranquios más comúnmente capturadas en pesquerías comerciales del estado de Sucre, Venezuela. Mustelus higmani (a), Mustelus canis (b), Hypanus guttatus (c) y Hypanus americanus (d).

Results showed that for M. higmani and M. canis, male specimens were largely more dominant than females and that there were sex-related differences in their size structures. Discrepancies between sexes may be related to differences in certain biological variables, such as growth parameters (Cortés et al. 2012). Previous studies for these 2 species indicate that growth rates and maximum sizes are different between sexes (Conrath et al. 2002, Carrillo 2019). Moreover, spatial and sexual segregation in elasmobranchs could be the result of the philopatric behavior exhibited by females, which tend to move less distances compared to males (Heist 2012).

With respect to the conservation assessments, 7 of the species recorded in the study area (M. higmani, C. limbatus, C. falciformis, I. oxyrinchus, A. superciliosus, P. glauca, and A. narinari) are listed as vulnerable at the national level. Tavares (2015) stated that the culprits of this risk include long-term and uncontrolled intensive fishing, capturing high proportions of sexually immature specimens, and degrading essential habitats. In a global context, different elasmobranch conservation strategies have been proposed, such as implementing fishing bans for certain fishing areas, regulating fishing gear, and establishing fishing quotas (Shiffman and Hammerschlag 2016); however, these measures have not been fully effective in most regions. More recently, the creation of marine protected areas (MPAs) to preserve elasmobranchs previos sobre estas 2 especies han indicado la existencia de diferencias entre sexos con respecto a las tasas de crecimiento y las tallas máximas (Conrath et al. 2002, Carrillo 2019). Sin embrago, la segregación espacial y por sexos en los elasmobranquios también puede tener su origen en el comportamiento más filopátrico exhibido por parte de las hembras, las cuales tienden a desplazarse espacialmente en menor grado en comparación con los machos (Heist 2012).

En cuanto a las evaluaciones de conservación, 7 de las especies registradas en el área de estudio (M. higmani, C. limbatus, C. falciformis, I. oxyrinchus, A. superciliosus, P. glauca y A. narinari) se encuentran en condición vulnerable a nivel nacional. Según Tavares (2015), las causas abarcan la pesca intensiva llevada a cabo durante largos periodos de tiempo y sin ningún tipo de control, la captura de proporciones elevadas de ejemplares sexualmente inmaduros y la degradación de los hábitats esenciales. En el contexto global, se han propuesto diferentes estrategias para promover la conservación de elasmobranquios, como la prohibición de las capturas en determinadas zonas de pesca, las regulaciones de las características de los métodos de pesca y el establecimiento de cuotas de captura (Shiffman y Hammerschlag 2016); sin embargo, estas medidas no han sido totalmente eficaces en la mayoría de las regiones. Más recientemente, la creación de áreas marinas protegidas con el propósito de preservar a los 
Marquez et al.: Elasmobranchs off Sucre State

and their habitats has increasingly attracted the attention of researchers and conservationists. Several examples of this conservation measure have been described and discussed in several studies (Garla et al. 2006, Knip et al. 2012).

In Venezuela, there is only one national fishing resolution to prohibit the fishing and marketing of C. falciformis, A. superciliosus, Carcharhinus longimanus, and species of the family Sphyrnidae (Gaceta Oficial de la República Bolivariana de Venezuela, 19 June 2012, no. 39.947). Nevertheless, most fishing regulations in the resolution are not being followed in most Venezuelan insular and coastal regions. The reasons for the neglect are diverse, but the main reason is the collapse of national institutions in charge of regulating and managing fishery resources that came after the country's economic, social, and political crisis in recent years (Tavares 2019). For now, an effort should be made to continue generating and supplementing information for the most important fishery resources; however, future advances in scientific research and conservation will depend, to a large extent, on the recovery of the country's political and economic stability.

\section{ACKNOWLedgments}

We thank the staff at the Fishery Resources Evaluation Lab in the Fisheries Biology Department, Instituto Oceanográfico de Venezuela, for their support during this study. We would also like to thank Hernán Mundarai, Luis Córdova, Fran Rivera, and other merchants at Sucre's fishing port and the municipal market of the city of Cumaná.

\section{REFERENCES}

Baum JK, Myers RA. 2004. Shifting baselines and the decline of pelagic sharks in the Gulf of Mexico. Ecol. Lett. 7(2): 135-145. https://doi.org/10.1111/j.1461-0248.2003.00564.x

Baum JK, Myers RA, Kehler DG, Worm B, Harley SJ, Doherty PA. 2003. Collapse and conservation of shark populations in the northwest Atlantic. Science 299(5605): 389-392. http://dx.doi.org/10.1126/science.1079777

Carrillo LAM. 2019. Edad y crecimiento de la viuda amarilla, Mustelus higmani (Springer y Lowe 1963), de la región nororiental de Venezuela durante el periodo agosto 2016-julio 2017 [BSc thesis]. [Boca de Rio (Venezuela)]: Universidad de Oriente; 68 pp.

Castellanos P, Varela R, Muller-Karger F. 2002. Descripción de las áreas de surgencia al sur del Mar Caribe examinadas con el sensor infrarrojo AVHRR. Mem. Fund. La Salle Cienc. Nat. 154: $55-76$.

Cervigón F, Alcalá A. 1999. Los peces marinos de Venezuela: Tiburones y rayas. Vol. 5. Estado Nueva Esparta (Venezuela): Fundación Museo del Mar; 230 pp.

Clarke S, Milner-Gulland EJ, Bjørndal T. 2007. Social, economic, and regulatory drivers of the shark fin trade. Mar. Resour. Econ. 22(3): 305-327. https://doi.org/10.1086/mre.22.3.42629561

Compagno LJV. 2002. Sharks. In: Carpenter KE (ed.), The living Marine Resources of the Western Central Atlantic: Species identification guide for fishery purposes, Special Publication No. 5. Rome: FAO; p. 358-505. elasmobranquios y sus hábitats esenciales está llamando cada vez más la atención de los investigadores y conservacionistas. Diversos ejemplos de esta medida de conservación han sido descritos y discutidos a través de varios estudios (Garla et al. 2006, Knip et al. 2012).

En el caso de Venezuela, se cuenta únicamente con una regulación pesquera que prohíbe la pesca y la comercialización de C. falciformis, A. superciliosus, Carcharhinus longimanus y las especies de la familia Sphyrnidae (Gaceta Oficial de la República Bolivariana de Venezuela 19 de junio de 2012, no. 39.947). No obstante, la mayoría de las normas establecidas en dicha resolución no se cumplen en casi ninguna de las regiones costeras e insulares venezolanas. Las razones del incumplimiento son variadas, pero la principal tiene que ver con el colapso de las instituciones del estado encargadas de la administración y el ordenamiento de los recursos pesqueros durante los últimos años como consecuencia de la crisis económica, social y política del país (Tavares 2019). Por el momento, se deberá hacer un esfuerzo para continuar generando y complementando información sobre los recursos pesqueros más importantes; sin embargo, los avances futuros en materia de investigación científica y conservación dependerán, en gran medida, de la recuperación de la estabilidad económica y política del país.

\section{Agradecimientos}

Se agradece al personal del Laboratorio de Evaluación de Recursos Pesqueros del Departamento de Biología Pesquera del Instituto Oceanográfico de Venezuela su apoyo en la realización de la presente investigación. También un agradecimiento a Hernán Mundarai, Luis Córdova, Fran Rivera y demás comerciantes del puerto pesquero de Sucre y el mercado municipal de Cumaná.

Conrath CL, Gelsleichter J, Musick JA. 2002. Age and growth of the smooth dogfish (Mustelus canis) in the northwest Atlantic Ocean. Fish. Bull. 100(4): 674-682.

Cordovés M, Ron E, Tavares R. 2010. Composición de las capturas comerciales de la raya látigo-hocicona, Dasyatis guttata (Bloch \& Schneider, 1801), procedentes de la pesca artesanal en la Isla de Cubagua, Venezuela. Gulf Caribb. Fish. Inst. 62: 567-570.

Cortés E, Brooks EN, Gedamke TD. 2012. Population dynamics, demography and stock assessment. In: Carrier JC, Musick JA, Heithaus MR (eds.), Biology of Sharks and their Relatives. 2nd ed: CRC Press, New York; p. 453-486. https://doi.org/10.1201/b11867

Dulvy NK, Fowler SL, Musick JA, Cavanagh RD, Kyne PM, Harrison LR, Carlson JK, Davidson LNK, Fordham SV, Francis MP, et al. 2014. Extinction risk and conservation of the world's sharks and rays. Elife 3:e00590. https://doi.org/10.7554/eLife.00590

Gaceta Oficial de la República Bolivariana de Venezuela. 2012 Jun 19. Resolución mediante la cual se dictan las normas técnicas de ordenamiento para regular la captura, intercambio, distribución, comercio y transporte de tiburones, No. 39.947. Caracas (Venezuela): Ministerio del Poder Popular para la Agricultura y Tierras. 
Garla RC, Chapman DD, Shivji MS, Wetherbee BM, Amorim AF. 2006. Habitat of juvenile Caribbean reef sharks, Carcharhinus perezi, at two oceanic insular marine protected areas in the southwestern Atlantic Ocean: Fernando de Noronha Archipielago and Atol das Rocas, Brazil. Fish. Res. 81(2-3): 236-241. http://doi.org/10.1016/j.fishres.2006.07.003

Heist EJ. 2012. Genetics of sharks, skates, and rays. In: Carrier JC, Musick JA, Heithaus MR (eds.), Biology of Sharks and their Relatives. 2nd ed: CRC Press, New York; p. 487-504.

[IUCN] International Union for Conservation of Nature. 2019. The IUCN red list of threatened species; [accessed 2019 May]. https://www. iucnredlist.org

Knip DM, Heupel MR, Simpfendorfer CA. 2012. Evaluating marine protected areas for the conservation of tropical coastal sharks. Bio. Con. 148(1): 200-209. http://doi.org/10.1016/j.biocon.2012.01.008

Kolmann MA, Elbassiouny AA, Liverpool EA, Lovejoy NR. 2017. DNA barcoding reveals the diversity of sharks in Guyana coastal markets. Neotrop. Ichthyol. 15(4): 1-7, e170097. http://dx.doi.org/10.1590/1982-0224-20170097

McEachran JD, de Carvalho MR. 2002. Batoids fishes. In: Carpenter KE (ed.), The living marine resources of the Western Central Atlantic: FAO Species identification guide for fishery purposes. Rome: FAO and American Society of Ichthyologists and Herpetologists Special Publication No. 5; p. 507-589.

Margalef R. 1969. El ecosistema pelágico del mar Caribe. Mem. Soc. Cienc. Nat. La Salle 29(82): 5-36.

Nelson JS, Grande TC, Wilson MVH. 2016. Fishes of the World. 5th ed. Hoboken (NJ): John Wiley \& Sons; 707 pp. https://doi.org/10.1002/9781119174844
Novoa D, Mendoza J, Marcano L, Cárdenas J. 1998. El Atlas Pesquero Marítimo de Venezuela. Caracas: MAC/SARPA; 197 pp.

Shiffman DS, Hammerschlag N. 2016. Shark conservation and management policy: a review and primer for non-specialists. Anim. Conserv. 19(5): 401-412. https://doi.org/10.1111/acv.12265

Shing CCA. 2006. Shark fisheries of Trinidad and Tobago: A National Plan of Action. Gulf Caribb. Fish. Inst. 57: 205-213.

Stevens JD, Bonfil R, Dulvy NK, Walker PA. 2000. The effects of fishing on sharks, rays, and chimaeras (Chondrichthyans), and the implications for marine ecosystems. ICES. J. Mar. Sci. 57(3): 476-494. https://doi.org/10.1006/jmsc.2000.0724

Tavares R. 2015. Condrictios. In: Rodríguez JP, Garcia-Rawlins A, Rojas-Suarez F (eds.), Libro Rojo de la Fauna Venezolana. 4th ed. Caracas (Venezuela): PROVITA y Fundación Empresas Polar; p. 264-277.

Tavares R. 2019. Estado actual de los tiburones y rayas (Pisces: Elasmobranchii) en el Mar Caribe venezolano: biodiversidad, pesca y conservación. Cienc. Pesq. 27(2): 33-55.

Tavares R, Sanchez L, Medina E. 2010. Artisanal fishery and catch structure of the smalleye smooth-hound shark, Mustelus higmani (Springer \& Low, 1963), from the northeastern region of Venezuela. Proc. Annu. Gulf Caribb. Fish. Inst. 62: 446-449.

Weigmann S. 2016. Annotated checklist of the living sharks, batoids and chimaeras (Chondrichthyes) of the world, with a focus on biogeographical diversity. J. Fish Biol. 88(3): 837-1037. https://doi.org/10.1111/jfb.12874

Zar J. 1996. Biostatistical analysis. 3rd ed. Upper Saddle River (NJ): Prentice Hall; 994 pp.

Received June 2019, accepted October 2019. 